\title{
Análisis y tendencias migratorias en la región del Biobío (Chile) entre 1982 y 2002. Aplicación desde el modelo gravitacional
}

\section{Analysis and migration trends in the Biobío region (Chile) between 1982 and 2002. Application from the gravity model}

\author{
Francisco Maturana-Miranda* \\ Andrés Rojas-BötTner* \\ David Poblete-López**
}

\begin{abstract}
The process of internal migration is analyzed in the Biobio Region; this yields a process of spatial redistribution tending to the polarization of the space towards the regional capital "Concepción Metropolitano" and towards other adjacent municipalities. Similarly, it is observed a strong migratory attraction to the provincial capitals of Chillan and Los Angeles, but confined to their hinterland. Additionally, the profile of the migrant who moves from these cities to the regional capitals is characterized by a high level of education, while the migrants from neighboring municipalities to provincial capitals are mainly young people with low schooling levels.
\end{abstract}

Keywords: internal migration, Biobio region, gravity model.

\section{Resumen}

El proceso de migraciones internas en la región del Biobío expresa un proceso de redistribución espacial que tiende a la polarización del espacio hacia la capital regional -Concepción metropolitano-y a las comunas contiguas a ésta. De igual manera, se observa una fuerte atracción migratoria de las capitales provinciales pero circunscrita a su hinterland. Además, se determina que el perfil del migrante desde estas ciudades hacia la capital regional se caracterizaría por una alta educación, a diferencia de los migrantes de las comunas aledańas a las capitales provinciales, que se compondrían más bien por población joven con bajos grados de escolaridad.

Palabras claves: migraciones internas, región del Biobío, modelo gravitacional tado.cl

* Departamento de Geografía, Universidad Alberto Hurtado, Chile. Correo-e: fmaturana@uahur-

** Instituto Chileno de Estudios Municipales, Universidad Autónoma de Chile. Correo-e: arojas@ichem.cl

*** Escuela de Ingeniería Civil, Universidad de Valparaíso, Chile. Correo-e: david.poblete@uv.cl 


\section{Introducción}

Los fenómenos migratorios en América Latina tienen antecedentes históricos. En especial desde mediados del siglo XIX, varios países de la región se convirtieron en receptores de numerosos contingentes de personas en busca de oportunidades, arribando principalmente a países como Argentina, Uruguay y Brasil. Dicho proceso disminuyó paulatinamente conforme avanzaba el siglo XX, hasta revertirse la tendencia en el último tercio, convirtiéndose América Latina en expulsora de población, principalmente hacia Estados Unidos y Europa, y también de manera interna en la región, como los flujos entre Colombia y Venezuela, o la movilidad de las áreas andinas hacia Argentina y Chile, entre otros (Martínez, 2008; Solimano, 2008). En cuanto a este último país, de manera reciente se ha convertido en un territorio atractivo para la migración latinoamericana, especialmente entre los países fronterizos (Perú, Bolivia y Argentina), aun cuando en términos relativos todavía los migrantes extranjeros representen apenas $2 \%$ de la población (INE, 2012). Mucho más relevantes para el país han sido las migraciones internas, entendidas como "aquella migración en que tanto el lugar de origen como el de destino están situados en el mismo país" (INE, 2007b: 1). Dentro de los principales procesos asociados se encuentran las grandes oleadas migratorias de finales del siglo XIX y comienzos del XX de trabajadores del centro y sur del país hacia el norte salitrero debido a la gran demanda de mano de obra en las calicheras. Posteriormente, entre las décadas de 1940 y 1960 se produjeron grandes desplazamientos de población desde el campo a la ciudad, especialmente hacia la capital Santiago y en menor medida grandes urbes como Valparaíso y Concepción (Hurtado, 1966).

Estas migraciones internas, al igual que en el resto de América Latina, han resultado fundamentales para entender los procesos de urbanización (Lattes, 2001). En la actualidad la magnitud de los flujos migratorios es relativamente baja, no obstante, son relevantes en términos de recomposiciones espaciales. De hecho, los diferentes ritmos de crecimiento de población entre regiones en el país se explican principalmente por el factor migratorio, ya que las diferencias en términos del crecimiento vegetativo en el país son menores entre los distintos territorios (Rodríguez y González, 2006). Sumado a lo anterior, se definen nuevos perfiles migratorios, con respecto a los puntos de desplazamiento (urbano-urbano), así como a las características de los sujetos; elementos que no han sido suficientemente estudiados en sus contextos específicos.

Los factores que desencadenan el proceso migratorio interno, según Rodríguez y Busso (2009), han estado básicamente asociados a motivaciones económicas, sociales y culturales. Cada una de ellas, con sus espe- 
cificidades, se relacionan con oportunidades, en términos de empleo, calidad de vida, acceso a educación, territorialidad o arraigo del lugar y redes sociales, entre otros. Por otro lado, Zelinsky (1971) en su trabajo conceptual sobre la hipótesis migratoria, plantea fases asociadas con los niveles de complejidad de una sociedad. Por lo tanto, en una etapa inicial, los movimientos serían más bien incipientes, y a medida que los estadios se van sucediendo, las migraciones campo-ciudad pueden tomar relevancia. Posteriormente, éstas cobrarían un rol secundario, predominando ahora los procesos de movilidad en territorios metropolitanos, donde los avances tecnológicos y las mejoras en el transporte invisibilizan ciertos procesos migratorios, bajo la forma de desplazamientos cotidianos por trabajo, estudio, diversión y otros.

El proceso de migraciones internas contribuye en la dinámica de ciertos espacios urbanos, especialmente a partir de una serie de desafíos en temas como vivienda (incluyendo la vivienda social), transporte y empleo (Hidalgo y Zunino, 2011; Borsdorf et al., 2008; Hall y Pain, 2006). Éstos irán configurando lo que Veltz (2005) ha denominado verdaderos archipiélagos metropolitanos, donde los agentes inmobiliarios toman un rol relevante (Hidalgo y Janoschka, 2014). Todo lo anterior debe entenderse bajo una lógica de competencia y marketing urbano de las ciudades, las cuales buscan ser cada día más atractivas en cuanto a su posicionamiento en el sistema territorial (Precedo et al., 2010), lo cual en cierta manera fortalecería el proceso migratorio.

Desde el punto de vista de su aproximación, el estudio de las migraciones internas está asociado al proceso de interacción espacial, el cual resulta fundamental para comprender su dinámica en cuanto a la existencia de procesos de atracción, repulsión, irradiación y cooperación (Camagni, 2005; Pumain y Saint-Julien, 2001). En este marco, uno de los modelos que ha contribuido al estudio de estas migraciones corresponde al Modelo Gravitacional. Éste, de larga tradición, se construye en analogía a la Ley de Gravitación Universal de Newton, referida a la atracción de dos cuerpos en razón directa de su masa y en razón inversa de la distancia que los separa (Karemera, et al., 2000; Faura y Gómez, 2001; Curry, 1972). Su utilidad radica en la posibilidad de predecir la magnitud de flujos que ocurren entre dos lugares, para posteriormente comparar los flujos reales y los estimados por el modelo. De tal forma, es posible comprender las atracciones o evidenciar las resistencias a la interacción entre dos unidades (Pumain y Saint-Julien, 2001). Tras la Segunda Guerra Mundial, los aportes de Stewart (1948) y posteriormente otros autores como Olsson (1965) o Wilson (1971) se orientan a comprender los fenómenos migratorios y las movilidades domicilio-trabajo, cuyas bases irán contribuyendo a modificaciones del planteamiento original (Chen y 
Wang, 2008; Ceglowski, 2006; Roy, 2004; Anderson y Van Wincoop, 2003; Haynes y Fotheringham, 1984; Thomas y Huggett, 1980; Niedercorn y Bechdolt, 1969). Alan Wilson (1971), con su aporte a la maximización de la entropía, contribuyó de manera sustancial al perfeccionamiento del modelo. En este sentido, Camagni (2005: 86) plantea que "el principio de entropía permite determinar la configuración más probable de los desplazamientos", es decir, los flujos que se desarrollarán entre una zona i y una zona j, lo cual representa la estructura territorial de las localizaciones tanto laborales como residenciales de los individuos que componen el sistema. Así, diversas aplicaciones serán realizadas en el campo de las ciencias sociales en fenómenos y temáticas como la conmutación, migraciones, comunicaciones telefónicas, turismo, tráfico vehicular y aéreo, entre otros (Morley, et al., 2014; Groschet, et al., 2007; Fukunari y HyunHoon, 2006; Karemera et al., 2000).

En este sentido, las variaciones del modelo han sido sustanciales en función de la disciplina que se ha desarrollado. Por ejemplo, desde un punto de vista de la economía, un trabajo relevante ha sido la propuesta de Morley et al. (2014), vinculado al turismo y su análisis en función de la demanda entre diferentes países. También desde dicho enfoque se han estudiado las migraciones, destacado propuestas como la de Lewer y Van den Berg (2008), autores que aplican un análisis de las migraciones para 16 países de la Organización para la Cooperación y el Desarrollo Económico (OCDE) entre 1991 y 2000, valorando el potencial del modelo para generar escenarios hipotéticos de migraciones. Asimismo, Campaniello (2014) ha estudiado tal proceso, asociado además a las exportaciones para determinados países de la Unión Europea.

Otra área de estudio ha sido la migración vinculada a educación universitaria. Por ejemplo, Alm y Wintersb (2009) aplican el modelo para estudiar el caso del estado de Georgia (Estados Unidos), donde la distancia desempenaría un rol fundamental en el proceso migratorio.

Desde la econometría Durán-Fernández y Santos (2014) testean el parámetro de la distancia y su robustez en la aplicación del modelo. Lo anterior se aplica para el caso de México para los 31 estados y el Distrito Federal.

Otra perspectiva ha sido la desarrollada por Chen (2015), donde se analiza el rol de la distancia y su vínculo a la ley de potencias. Lo anterior aplicado para 29 regiones en China, compuesta cada una por la suma de la población urbana de sus ciudades, la distancia entre ellas y el volumen de carga (cantidad de mercancías transportadas en trenes) como función de gravedad. Así, observamos una abundante literatura que ha ido potenciando la formulación original (ecuación 1) con propuestas de mayor complejidad y robustez. 
Por su parte, en Chile ha existido un estudio sistemático del proceso migratorio, aunque a escalas diferenciadas. Un estudio pionero respecto al fenómeno de migraciones internas corresponde al realizado por $\mathrm{Di}$ Filippo y Bravo (1977), en el cual analizaron la distribución espacial y el fenómeno migratorio entre 1885 y 1970 para la población que componía las regiones de Chile. Los autores concluyeron que el proceso migratorio estaba fuertemente relacionado al efecto empleo, este último vinculado a la matriz exportadora e industrial de cada región. Por su parte, Raczynski (1982) analiza las causas de la migración rural-urbana, centrándose en factores como la disponibilidad de tierras y acceso a servicios en las zonas agrícolas. Posteriormente, Martínez (2008) analiza los procesos de redistribución espacial de la población en el país, confirmando la tendencia a la pérdida de población de las zonas extremas y su concentración en el centro, especialmente en la Región Metropolitana. Otros estudios recientes, como el de Rodríguez y González (2006) han planteado que entre los años 1970 y 2002 existió un importante proceso de desconcentración regional en términos migratorios. Por otra parte, el Instituto Nacional de Estadísticas (INE 2004, 2007b) plantea dos análisis, uno enfocado más bien a escala regional para el periodo 1992-2002 y un segundo análisis a esta misma escala con algunos elementos específicos a nivel comunal.

Desde otra perspectiva, centrando su análisis en la Región Metropolitana, Schiappacasse, et al. (2001) indican cómo ese territorio ha perdido peso en su atracción migratoria, a favor de ciudades o comunas de menor tamaño localizadas en otras regiones del país.

Además de lo ya indicado, es posible encontrar otros estudios (Rodrigo y Atienza, 2014; Busso, 2007; Aroca, 2004; Aroca, et al., 2001) en temáticas afines, como economía espacial, centrándose en mercados de trabajo, efectos sociodemográficos y pobreza, aunque a una escala de análisis comparativo entre espacios regionales.

Dado lo anterior y a pesar de los trabajos recién destacados, no son abundantes las investigaciones que han profundizado en los procesos migratorios internos entre comunas a una escala regional.

El presente artículo centra su análisis en las comunas de la Región del Biobío, localizada aproximadamente a $370 \mathrm{~km}$ al sur de la capital de Chile (figura 1). La región se caracteriza por una estructura económica con gran peso de las actividades forestales, fuertemente desarrolladas en los últimos 35 años; agropecuarias e industriales, destacando como factor relevante la concentración de población en torno a la capital regional Concepción, segundo centro urbano del país, con un área metropolitana compuesta por seis comunas.

Cabe destacar que la división político-administrativa de Chile está compuesta de manera jerárquica por regiones, provincias y comunas. Las 
ciudades en general están contenidas al interior de una comuna, salvo los tres grandes espacios metropolitanos del país: Santiago, Concepción y Valparaíso, más algunas capitales regionales o provinciales que por su crecimiento han desbordado los límites comunales.

La Región del Biobío está compuesta por cuatro provincias (figura 1): Concepción (capital Concepción), Nuble (capital Chillán), Biobío (capital Los Ángeles) y Arauco (capital Lebu), las cuales presentan algunas variaciones en su estructura productiva relevantes para entender el comportamiento migratorio. Si consideramos el número de puestos de trabajo (INE, 2002) como un indicador de la composición sectorial de su economía, la provincia de Concepción presenta una estructura diversificada, destacando los sectores comercial (19\%) e industrial (15\%). La provincia de Nuble, en cambio, presenta menor desarrollo industrial y un mayor peso relativo de las actividades agropecuarias (21\%), aunque un pequeño sector forestal (4\%). La provincia del Biobío a su vez presenta un mayor equilibrio entre las actividades agropecuarias (13\%) y las silvícolas (7\%), destacando además el número de empleos en las áreas de comercio (16\%), industria (15\%) y construcción (11\%). Finalmente, la provincia de Arauco presenta un muy relevante sector forestal, con más de $14 \%$ de los puestos de trabajo, a la vez que un sector agrícola relativamente menor (9\%) y proporcionalmente el mayor peso de las actividades pesqueras, lo que la convierte en una provincia con predominio de actividades primarias y en menor medida comerciales (14 por ciento).

En este artículo se plantearon como objetivos: 1. comprender las dinámicas migratorias y cambios en las estructuras espaciales en la Región del Biobío entre 1977 y 2002; 2. observar y analizar la atractividad y efectos barrera que se puedan estar generando en el espacio, y 3. aplicar el Modelo Gravitacional al estudio de las migraciones internas en Chile.

Esta investigación concluye que se está desarrollando un proceso de redistribución espacial en las migraciones internas efectuadas entre las comunas de la región, tendiendo a la polarización del espacio hacia la capital regional -Concepción metropolitano- y a las comunas contiguas a ésta. De igual manera, se prevé una fuerte atracción migratoria de las capitales provinciales Chillán y Los Ángeles, pero circunscrita a su hinterland. Finalmente, se estima que el perfil del migrante desde estas ciudades hacia la capital regional se caracterizaría por una alta educación, a diferencia de los migrantes de las comunas aledañas a las capitales provinciales, que se compondrían más bien por población joven con bajos grados de escolaridad. 


\section{Materiales y método}

Para llevar a cabo este estudio se consideraron cinco etapas:

\subsection{Periodo de análisis, flujo migratorio y confección de matrices}

Se analizaron tres periodos, correspondientes a 1977-1982, 1987-1992 y 1997-2002, en razón de la disponibilidad de datos censales. ${ }^{1}$ Posteriormente, se estableció el número de comunas en estudio. Actualmente la región del Biobío está conformada por 54 comunas (figura 1), sin embargo, por diferentes modificaciones en la división política administrativa del país y el área metropolitana de su capital regional, se procedió a trabajar con tres territorios ${ }^{2}$ (que componen 10 comunas) y las 44 comunas restantes. Los territorios son Gran Concepción, ${ }^{3}$ conurbación ChillánChillán Viejo (en adelante, Chillán) y Alto Biobío-Santa Bárbara (dado que la primera comuna fue creada en 2003 y no cuenta con datos desagregados).

Los flujos fueron extraídos a partir de la pregunta de los censos de población respecto del lugar de residencia cinco años antes de la fecha del censo. Así, para 1982 se interrogaba la residencia de 1977, mientras que para 1992 la de 1987 y en 2002 la de 1997. Las bases de datos fueron proporcionadas por el INE y manipuladas mediante el programa gratuito Redatam versión 5, R+SP. Con el número de comunas y flujos establecidos, se procedió a confeccionar para cada periodo una matriz de 47X47 con el programa gratuito y libre Calc, en el cual se ingresaron los flujos para cada par de comunas en los periodos señalados.

\subsection{Análisis de la tasa de migración interna}

Para realizar una correcta interpretación, se analizó la tasa de migración interna de cada comuna, entendida como la capacidad de atracción en relación con su saldo migratorio y la población existente en dichos territorios. Así se obtuvo una visión general del fenómeno de la migración

\footnotetext{
${ }^{1}$ Se debe destacar que el Censo de población realizado en 2012 fue declarado legamente inutilizable por los errores cometidos durante el proceso. Actualmente se prepara un nuevo levantamiento abreviado para el 2017.

${ }^{2}$ Dos de ellos por agrupación funcional y un tercero (Alto Biobío-Santa Bárbara) producto de la creación de la primera comuna, posterior a la disponibilidad de datos censales.

${ }^{3}$ Unidad metropolitana que comprende cinco comunas: Concepción, Chiguayante, San Pedro de la Paz, Talcahuano, Penco y Hualpén (ésta, creada en 2004 a partir de la Comuna de Talcahuano). Se decidió considerar esta agrupación de comunas ya que es la utilizada por el Instituto Nacional de Estadísticas de Chile, organización que generó los datos disponibles.
} 
interna para el periodo comprendido entre 1982 y 2002. Para tal caso se aplicó la siguiente ecuación:

Ecuación 1

$$
\text { Tmi (i)=saldo } / \text { población } \times 1000
$$

Donde:

Saldo: corresponde a la resta entre las llegadas y las salidas.

Población: La población total residente en las comunas para el periodo.

\section{3. Aplicación del Modelo Gravitacional}

Desde su formulación más sencilla a una compleja, el Modelo Gravitacional consiste en poder estimar un valor de flujo teórico entre dos pares de comunas (en este caso), que posteriormente debe ser contrastado con un valor real, para así obtener un valor residual y observar la atracción y efectos barrera que se generan en el espacio (Pumain y Saint-Julien, 2001). La formulación básica del modelo está dada por:

\section{Ecuación 2}

Donde

$$
F i j=k * P i * P j * D i j^{-\alpha}
$$

Fij = es el flujo teórico entre dos territorios i e j.

$k=$ una constante

$\mathrm{Pi}=$ Población del territorio $\mathrm{i}$

$\mathrm{P} \mathbf{j}=$ Población del territorio $\mathrm{j}$

$\mathrm{Dij}^{\alpha}=$ Distancia entre los territorios $\mathrm{i} \mathrm{y} \mathrm{j}$

$\alpha=$ exponente que determina la influencia de la distancia en el proceso a modelar.

Respecto a la ecuación, es necesario analizar y considerar el significado en tres de sus componentes. El primer elemento corresponde al parámetro $k$, el cual es una relación entre el volumen de flujos y la población de los territorios, es decir, la tasa de movilidad asociada a la dificultad de vencer la distancia (Pumain y Saint-Julien, 2001). La particularidad que hace aplicable el Modelo Gravitacional a las ciencias sociales radica en que el parámetro k no será una constante para todos los fenómenos, sino determinada por la matriz de flujos reales que ocurren en el sistema (Thomas y Huggett, 1980).

Un segundo elemento es referente a la multiplicación de las masas. La cantidad de población de los territorios está determinando la capacidad 
de emisión o atracción de un lugar, la cual supone que cada individuo de un territorio puede establecer una interacción con otro habitante de un espacio diferente.

Finalmente, la distancia es otro elemento a destacar, puesto que mientras ésta sea mayor, menor será la interacción espacial (Thomas y Huggett, 1980). Además, es necesario considerar un factor que pueda estimar la influencia que tendrá la distancia en los flujos entre comunas. Por lo general, para modelos físicos de cualquier naturaleza, se estima que la influencia de un fenómeno disminuye exponencialmente al cuadrado con la distancia, es decir con un alpha igual a 2. En este caso, alpha puede tomar valores cercanos a 2, y será igual para todos los flujos del sistema. Otra mirada que se puede dar a alpha es que adquiera valores distintos para cada comuna, dependiendo de factores físicos, económicos o sociales; los llamados efectos barrera que pueden estar relacionados con sistemas de transporte deficitarios, la no existencia de carreteras, dificultades para la migración, conflictos sociales, bélicos, falta de oportunidades laborales, etcétera.

En este artículo las distancias entre comunas se determinaron como puntos de origen y destino de los centros urbanos de mayor población de cada una. ${ }^{4}$ Fueron extraídas de la Dirección de Vialidad del Ministerio de Obras Públicas de Chile, la cual entrega la distancia entre todos los centros urbanos del país. ${ }^{5}$

Respecto a la ecuación y modelo empleado, se determinó aplicar el modelo Entropía o tipo Wilson que entrega un correcto ajuste para este tipo de estudios (Camagni, 2005; Pumain y Saint-Julien, 2001). ${ }^{6}$ Éste considera la probabilidad de ocurrencia de los distintos escenarios de movilización entre comunas, conocidos los valores de oferta y demandas (Oi y $\mathrm{Dj}$ ) y respecto a parámetros y variables utilizadas, lo que busca es

\footnotetext{
${ }^{4}$ Dada la variación del modelo utilizado en este artículo, la población de cada comuna, como se analizará, no fue considerada.

${ }^{5}$ Esta manera de calcular las distancias también es sugerida por Thomas y Huggett (1980). Las distancias entre ciudades fueron extraídas de las distancias calculadad con el Sistema de Información Geográfica ArcGis 10 (2013).

${ }^{6}$ Como todo modelo, se debe considerar que solamente será una representación simplificada de la realidad, por lo tanto no se pretende que los flujos calculados a partir del Modelo Gravitacional se ajusten perfectamente a los valores medidos por el censo, pero sí que el modelo pueda representar los distintos procesos e interacciones en el territorio para entender los flujos que se desarrollan entre las distintas comunas. Además, se debe considerar que existen otras versiones del modelo posibles de aplicar. Se seleccionó la indicada puesto que diversos autores han demostrado su efectividad. Sin embargo, se testeó la versión Atracción-Emisión, que considera la suma de los flujos modelados de emisión y de atracción desde cada ciudad de manera iterativa, de forma que la inmigración y la emigración total a cada comuna sean iguales a los valores observados. Lamentablemente no entregó buenos ajustes y se descartó.
} 
maximizar la probabilidad de ocurrencia de los estados internos del sistema. $^{7}$

\section{Ecuación 3}

Donde:

$$
T i j=O i A i D j B j e^{-\beta c i j}
$$

Tij: flujo entre comuna i y comuna $j$

Oi: Cantidad de trabajadores viviendo en ciudad i

Dj: Cantidad de puestos de trabajo en comuna $j$

$\beta$ : factor de calibración del modelo.

Cij: costo de viaje entre i y j (distancia).

Además se debió considerar que:

$$
\begin{aligned}
& A_{i}=1 /\left[\sum_{j}^{n} B_{j} D_{j} e^{-\beta c_{i j}}\right] \\
& B_{j}=1 /\left[\sum_{j}^{n} A_{i} O_{i} e^{-\beta c_{i j}}\right]
\end{aligned}
$$

Para determinar los factores Ai y Bj se requiere un proceso de iteración, es decir, otorgándole un valor inicial a alguno de ellos para luego calcular el siguiente y se vuelve a hacer la misma operación hasta que los cambios entre iteraciones sean lo suficientemente pequeños. El parámetro beta multiplica a la variable de costos de viaje dentro de la exponencial y sirve como un factor de calibración del modelo y no tiene ningún significado físico. El factor del costo de viaje Cij, en el caso de este modelo simplificado, se asume como la distancia entre las distintas comunas. Este costo se puede estimar también usando costos monetarios (por ejemplo, valorando el combustible usado para viajar entre comunas) u otros costos indirectos (tiempos de viaje, peajes o tarifas en autopistas, dificultad de movimiento si se eligen caminos rurales en mal estado o autopistas que permiten gran velocidad, etcétera).

Para aplicar el modelo en cuestión se utilizaron matrices enunciadas en la metodología y se aplicaron las ecuaciones descritas con el programa Matlab versión R2012b. 


\subsection{Cálculo de los valores residuales a partir de la matriz de flujos reales y los teóricos obtenidos con el modelo}

Una vez obtenidos los valores teóricos a partir de la aplicación del modelo en cuestión, fue posible calcular los valores residuales y así observar la atracción y efectos barrera en la interacción migratoria de las comunas. Los valores residuales se obtuvieron según la siguiente ecuación:

\section{Ecuación 4}

Donde:

$$
F R i j=F O i j-F E i j
$$

FR $\mathrm{ij}=$ Flujo residual entre una zona $\mathrm{i}$ y otra $\mathrm{j}$.

FO $\mathrm{ij}$ = Flujo real u observado entre una zona $\mathrm{i}$ y otra $\mathrm{j}$.

FE ij = Flujo teórico entre una zona i e j calculada a partir del modelo.

\subsection{Análisis del perfil de edad y educación de los migrantes}

Finalmente, para complementar el análisis planteado, se procedió a analizar las edades de los migrantes, focalizándose en la población entre 15 y 40 años, que fue la que presentó mayor proporción de movimiento. Además, se estudió el tipo de educación, la cual se agrupó en población con estudios básicos, medios y universitarios. Se excluyó el tipo de formación técnico-profesional puesto que su participación era mínima. Por motivo de mejor ajuste del modelo, extensión de los datos y la importancia en el área de estudio, ambos análisis se focalizaron en el periodo 19972002 en los territorios del Gran Concepción, conurbación Chillán y comuna de Los Ángeles. Los datos fueron extraídos de la base censal del INE, a través del programa Redatam.

\section{Resultados}

\subsection{Análisis de la tasa de migración interna}

$\mathrm{Al}$ analizar la tasa de migración interna se observa que 27,6\% de las comunas en estudio presentan un valor positivo en el periodo 1982-2002. Esto da cuenta de los fuertes contrastes que se generan en el espacio regional (figura 1), puesto que la mayoría de las comunas se constituyen en expulsoras. Los flujos migratorios muestran tendencias esperables: las comunas con mayor cantidad de población son las que ejercen mayor captación, destacando la capital regional Concepción y dos capitales provinciales, Chillán y Los Ángeles. La otra capital provincial presente en la región, Lebu (provincia de Arauco, territorio número 4 en la figura 1) 


\section{Figura 1}

\section{Área de estudio y tasa de migración interna entre 1982 y 2002}

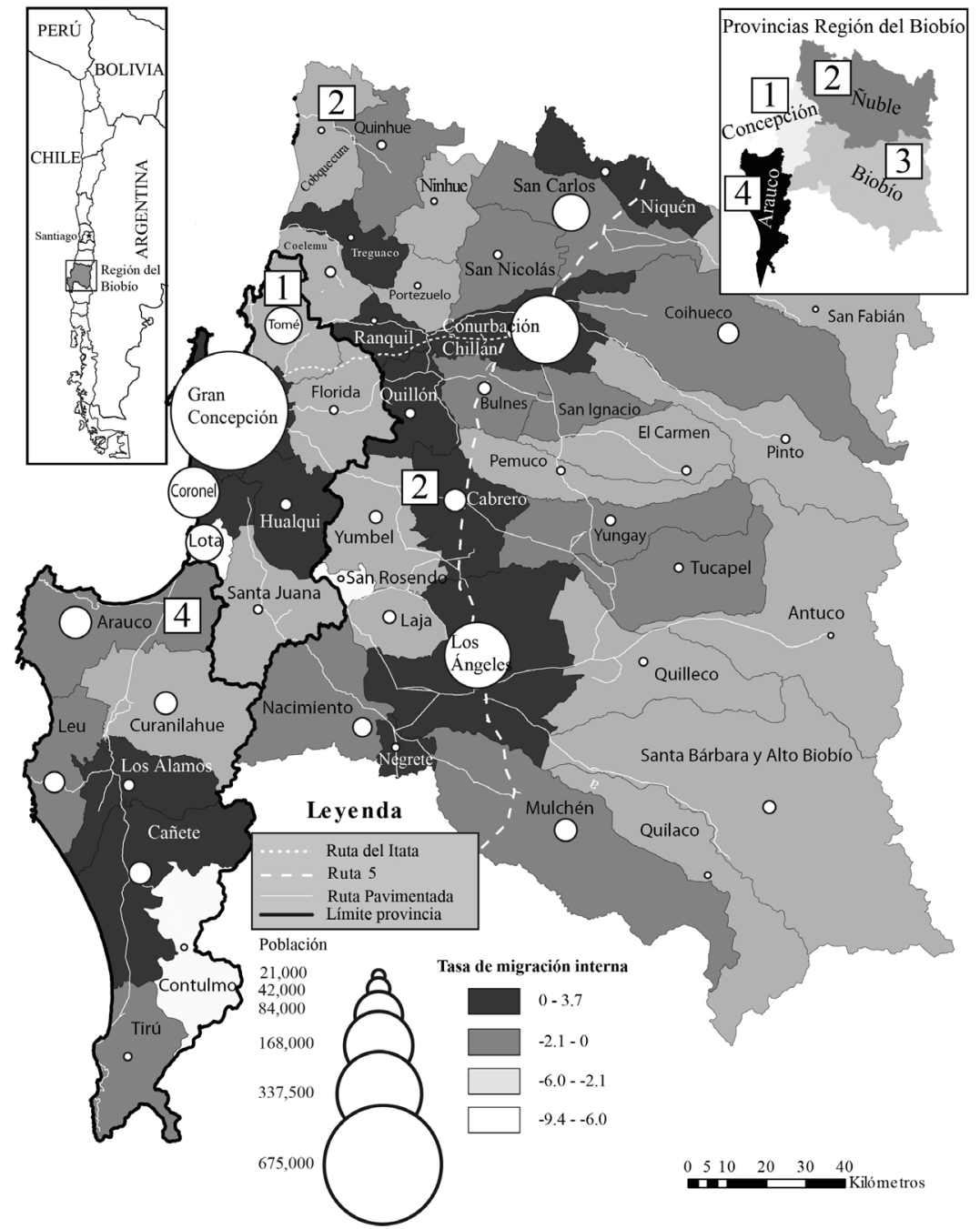

La localización de los círculos está dada por centroides de cada comuna y no corresponden necesariamente a su localización geográfica Los territorios insulares de la región fueron suprimidos de la cartografia.

Fuente: elaboración propia a partir de INE, 1982, 1992 y 2002.

presenta una negativa tasa de migración y por ello no forma parte del grupo descrito.

Si bien la tendencia es la expulsión de población en las comunas de menor cantidad de población, emergen algunas excepciones al respecto: por ejemplo, Los Álamos y Cañete (provincia de Arauco), especialmente la primera, bordeando una tasa de 2,3. Otra comuna relevante correspon- 
de a Negrete, que presenta una tasa de 3,6, que si bien se trata de bajos volúmenes (fundamentalmente desde Los Ángeles y Nacimiento), inciden de manera significativa en términos relativos, debido a la baja cantidad de población que ostenta dicho territorio. La atracción de Negrete se explica por una expansión urbana funcional de la ciudad de Nacimiento en el borde del límite administrativo con la Comuna de Negrete, lo que genera una situación muy particular, ya que la expansión urbana de Nacimiento en términos estadísticos es la de Negrete. El auge forestal y la instalación de una importante planta de celulosa y papel son un factor relevante en este proceso.

Otros casos a destacar son las comunas de Hualqui $(2,3)$ y Coronel $(2,4)$, que dada su cercanía a Concepción (mayor centro urbano de la región) se presentan como atractivas para el desarrollo inmobiliario de costo medio y bajo.

Otra característica relevante de las comunas que presentaron una tasa de migración interna positiva corresponde a su localización espacial. En efecto, la figura 1 expone cómo varios territorios en torno a la ruta 5 (principal ruta de cuatro vías que atraviesa parte importante del país) presentaron una tasa de positiva, lo cual evidenciaría la importancia de dicho eje. Además, en torno a éste se articulan las dos capitales provinciales de Chillán y Los Ángeles, generando una importante atractividad en las comunas contiguas en forma de una diagonal norte-sur (figura 1).

No obstante lo anterior, el sector este de la región (donde se localiza la cordillera de Los Andes) y algunas zonas del borde costero evidenciaron una tasa negativa. Entre las comunas con valores más bajos, destaca San Rosendo con -9,3 y Lota -6,0. La primera, vinculada a la pérdida de actividades relevantes, como el servicio ferroviario, y la segunda consecuencia de un declive económico relacionado con el fin de la minería carbonífera (Sandoval, 2011; Rodríguez y Medina, 2011), y planes de reconversión hacia al turismo que fracasaron. En similar situación se encuentran comunas como Contulmo -7,6, San Fabián -4,2 y Cobquecura $-4,1$, consecuencia de la baja rentabilidad de los cultivos agrícolas tradicionales y la expansión forestal.

\section{2. Aplicación del Modelo Gravitacional}

La aplicación del modelo arroja resultados bastante interesantes en cuanto al ajuste. En el cuadro 1 es posible observar los parámetros obtenidos y apreciar el coeficiente de determinación en los años aplicados. Este último para el modelo Wilson o de Entropía en el 2002 es bastante robusto, alcanzando $73,7 \%$ de ajuste. Esto no es así para los años anteriores, cuyo menor resultado alcanzan solamente 59,4 por ciento. 
Estas diferencias en los ajustes se pueden deber a distintas causas: calidad de los datos originales, cantidad de datos para el análisis, procesos reales que no son tomados en cuenta en el modelo, etcétera.

El primer ejemplo aplica para cualquier tipo de modelación, ya que, si los datos disponibles no son del todo confiables o hay una razón de peso para suponer que no son del todo exactos, los resultados que arroje el modelo en cuestión tendrán que analizarse tomando en cuenta las posibles restricciones en los datos. Esto puede ser cierto para los registros censales más antiguos, en donde la confianza en el proceso de recopilación puede no ser tan alta como en los últimos años.

Siguiendo la misma lógica, en algunos casos el hecho de que existan pocos datos puede hacer más débil el proceso de calibración de los modelos. Para este estudio no es el caso, pero en general un modelo con pocos datos será menos confiable y sus resultados, más inciertos. Esto no quiere decir que el modelo entregue resultados incorrectos, pero su análisis deberá tomar en cuenta estas restricciones.

Por último, una explicación posible para una calibración insatisfactoria para un modelo, es que éste mismo, en su concepción y desarrollo, no considere ciertos procesos que sí ocurren en la realidad. Esta explicación también es intrínseca de los procesos de modelación, los cuales son simplificaciones de la realidad y siempre se tomarán supuestos que se dan por hecho de manera de entender ésta con base en modelaciones.

\section{Cuadro 1 \\ Parámetros y resultados del Modelo Gravitacional de Entropía tipo Wilson}

\begin{tabular}{llr}
\hline & $R 2$ & Parámetro beta \\
\hline $1977-198$ & 59.4 & 0,0170 \\
$1987-1992$ & 65.3 & 0,0159 \\
$1997-2002$ & 73.7 & 0,0157 \\
\hline
\end{tabular}

Fuente: elaboración propia.

En este caso, los valores del parámetro beta sirven para ajustar el modelo al mejor factor del coeficiente de determinación, el cual no varía en más de $10 \%$ entre los periodos, lo que indica que el sistema no cambia radicalmente con el tiempo. Preliminarmente, al parecer, la disminución en el valor de beta podría implicar, dentro del razonamiento que se puede extraer del modelo, que las migraciones estarían aumentando entre comunas lejanas y que la distancia sería una variable cada vez menos 
restrictiva para migrar. No obstante, estudios posteriores podrían trabajar la hipótesis acá planteada.

Así, en el ajuste del modelo, algunas anomalías son posibles de estudiar a partir de la figura 2: en ella se presentan tres gráficos construidos para cada periodo de análisis. En el eje X se aprecian los flujos modelados o teóricos, y en el eje $\mathrm{Y}$ los observados o reales, de manera que los puntos que se encuentran bajo la recta están siendo subestimados con respecto a lo registrado en los censos. Por el contrario, los puntos sobre la recta del modelo están sobreestimados, es decir, el modelo predice mayor cantidad de personas (que realizan la migración) de lo observado. Así, las migraciones entre comunas que están bajo la recta poseen algún flujo de tipo preferencial y, por el contrario, las migraciones graficadas sobre la recta poseen algún efecto barrera, que no están siendo tomados en cuenta por el modelo (figura 2). En este sentido, se observó que algunos flujos eran de magnitud considerable en las tres matrices de análisis, destacando el envío de población desde la comuna de Lota hacia Coronel, tal como será explicado más adelante.

\section{Figura 2}

\section{Dispersión obtenida en la aplicación del Modelo de Entropía para los periodos 1977-1982, 1987-1992 y 1997-2002 entre flujos observados y modelados}
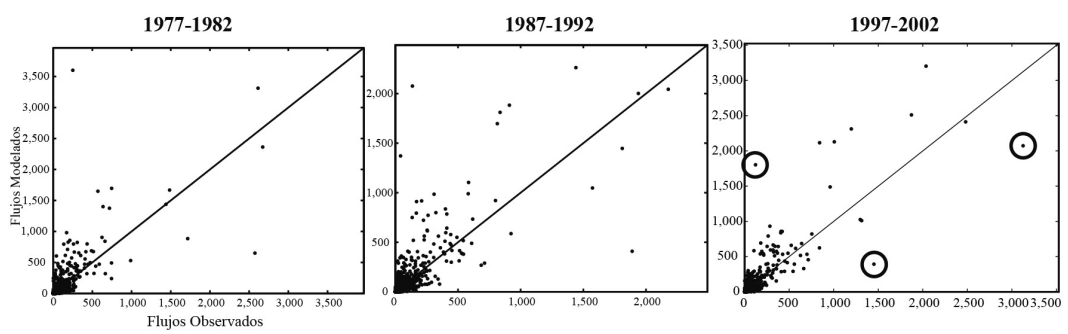

Fuente: elaboración propia a partir de INE, 1982, 1992 y 2002.

Posteriormente, ya con los resultados obtenidos por la aplicación del modelo, el siguiente paso fue constatar la atractividad y efectos barrera que se estaban generando en el espacio, es decir, el efecto que la distancia no estaría reflejando y donde otros elementos podrían estar incidiendo, como el costo de realizar el viaje según el medio de transporte, la atractividad laboral del lugar y el costo de vida, aspectos culturales, entre otros.

Una diferencia positiva en un caso de flujos observados y estimados podría significar que las personas tienden a migrar de mayor manera del territorio i hacia la j, es decir, observar flujos preferenciales en relación con el nivel de movilidad promedio y considerando el volumen de la 
población, según la ecuación utilizada (Pumain y Saint-Julien, 2001). Por el contrario, una diferencia negativa expresa una resistencia de las personas a migrar del territorio i hacia el j. Esta preferencia podría variar si, por ejemplo, se considera el perfil del tipo de migrante de acuerdo con sus tramos de edad u otra característica.

\subsection{Atractividad migratoria y efectos barrera: Las capitales provinciales Gran Concepción, Los Ángeles y Chillán}

A continuación, se analizan las preferencias migratorias y efectos barrera en los tres principales territorios de la región en el periodo 1997-2002, el cual es de mejor ajuste del modelo utilizado.

Respecto del Gran Concepción (localizado en la provincia de Concepción, territorio 1 en la figura 1), destacan las preferencias migratorias de las comunas de Los Ángeles $(+1,114)$, Tomé $(+529)$ y Laja $(+512)$ (figura 3). Otros casos relevantes fueron Florida $(+431)$, Curanilahue (+305), Lebu (+274) y Mulchén (+185). Se debe destacar que las preferencias migratorias hacia el Gran Concepción provienen de las comunas pertenecientes a todas las provincias, salvo la de Nuble (número 2 de la figura 1), en la cual es posible apreciar efectos barrera o baja atractividad. Algo similar ocurre respecto de las comunas próximas al área próxima a la cordillera de Los Andes.

Para el caso de Los Ángeles (localizada en la provincia del Biobío, número 3 de la figura 1), las comunas que desarrollan una mayor preferencia migratoria hacia este territorio están constituidas por dos grupos (figura 3). El primero, son los aledaños localizados al sur y este de la comuna, como Mulchén (+527), Nacimiento (+392), Santa Bárbara (+447), Quilleco (+361), Laja (+330) y Antuco (+182). Posteriormente, de manera más alejada, pero con mayor fuerza, destaca el caso del Gran Concepción $(+1,121)$, haciendo recíproca esta relación funcional preferencial en términos migratorios. Los efectos barrera están presentes al norte de Los Ángeles, cuya resistencia a la interacción queda reflejada con Chillán $(-167)$, la cual se constituiría en un ente gravitante en sus territorios aledaños.

En cuanto a Chillán (capital de la provincia de Nuuble), las preferencias migratorias están asociadas fundamentalmente a las comunas de su provincia, destacando los casos de Coihueco (+647), El Carmen (+478), San Carlos (+436), San Ignacio (+381) y Pinto (+355), entre otras. Si bien la provincia de Nuble presenta un carácter de cierto aislamiento relativo en comparación a las otras provincias de la región, ${ }^{8}$ el Gran Concepción

\footnotetext{
${ }^{8}$ Lo anterior expresado en sus deseos de independencia como región. Esto se materializó en agosto de 2015 con el envío al Congreso, por parte de la presidenta de la república, Michelle Ba-
} 
presenta una preferencia migratoria hacia Chillán (más bien unilateral), a diferencia de algunas comunas aledañas al Gran Concepción, como Hualqui (-79) o Coronel (-92), y también en el caso de Los Ángeles (-112). En síntesis, Chillán y su provincia se presentarían como una unidad funcional poco integrada a los demás núcleos de la región.

Finalmente, una comuna de la provincia de Arauco presenta un efecto barrera interesante a destacar: este corresponde a la comuna de Los Álamos (figura 1 para su localización y 3 para los flujos), cuya tasa de migración interna en el periodo destacó por un 2,3 (la quinta más alta de la región) y podría estar asociada al dinamismo en puestos de trabajo por la actividad forestal.

No obstante, el resto de las comunas presenta una expulsión de población relevante, lo cual llama a la reflexión el rol de la población indígena mapuche en dicho proceso. En efecto, si se analiza la cantidad de población mapuche que habita en las diferentes provincias, la provincia de Arauco está compuesta por $13 \%$ de población mapuche, mientras que el resto de los territorios no supera 2\% (INE, 2002). Esto se ve reflejado en que la dirección regional de la Corporación Nacional de Desarrollo Indígena (Conadi) ${ }^{9}$ se localiza en la comuna de Cañete, perteneciente a la provincia.

Al desagregar la migración solamente de población mapuche entre las provincias, el saldo hacia la provincia de Concepción resulta positivo, es decir, la población de esta etnia prefiere migrar hacia Concepción por sobre la provincia de Arauco. Si bien las magnitudes no son del todo relevantes, según el censo de 2002, 314 mapuches vivían en Arauco en 1997, mientras que en 2002 lo hacían en Concepción, lo cual corresponde $65 \%$ de los migrantes mapuches regionales del periodo. Por el contrario, el valor a la inversa es solamente 196.

\subsection{Preferencias migratorias y efectos barrera. Estructura y análisis regional}

Para complementar el análisis, se procedió a observar las principales preferencias (atractividad) y barreras migratorias a nivel regional. Lo anterior permite comprender dónde se refuerzan las interacciones y los lugares que presentan una resistencia a establecer vínculos migratorios.

chelet, del proyecto que crearía la región de Nuble, el cual actualmente se debate en el seno del Congreso Nacional.

${ }^{9}$ Organismo del Estado chileno que desarrolla toda la política pública en torno a los diferentes pueblos indígenas existentes en el país. 


\section{Figura 3 \\ Preferencias migratorias y efectos barrera en las migraciones recibidas por el Gran Concepción, conurbación Chillán y comuna de Los Ángeles, periodo 1997-2002}
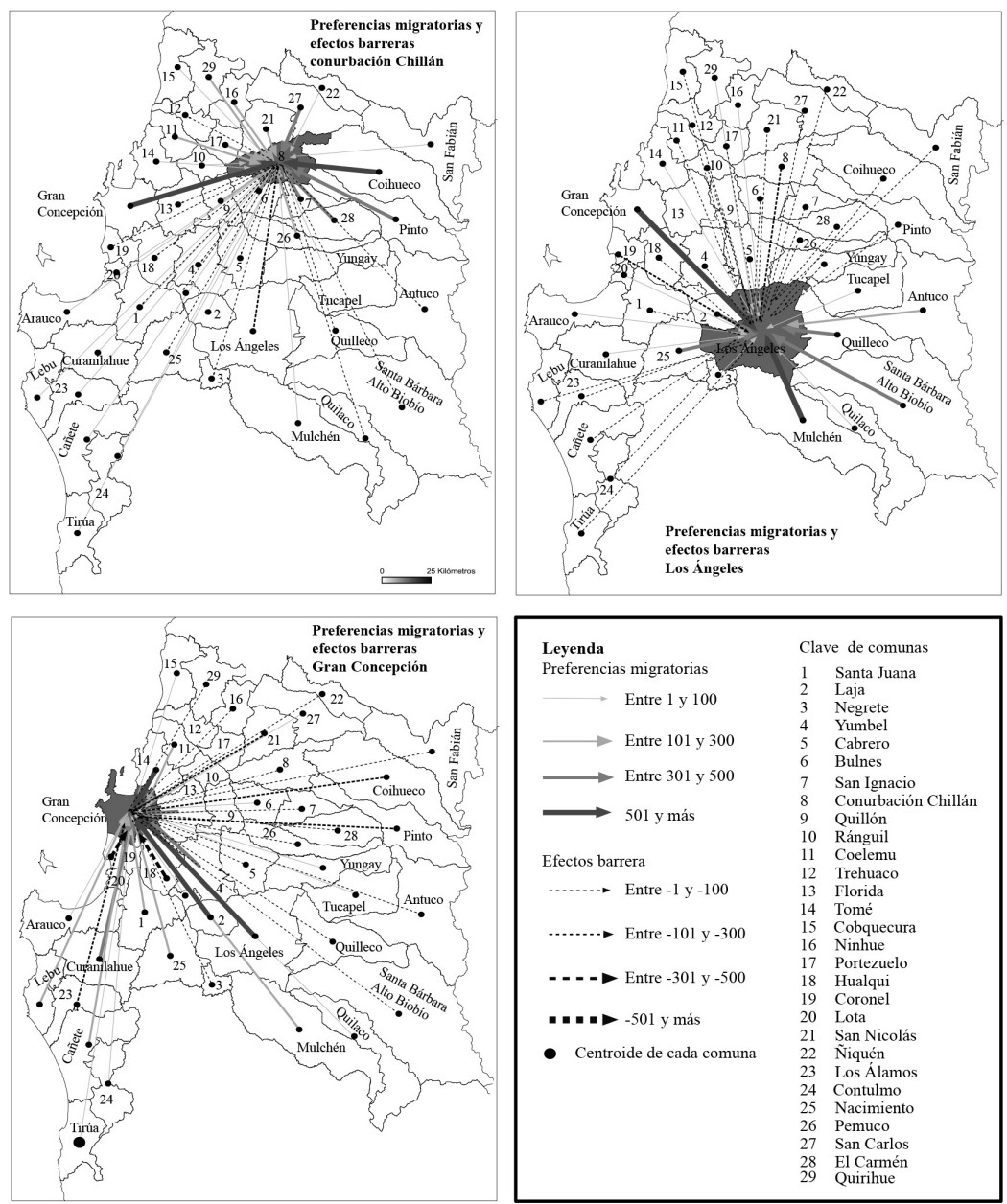

Fuente: elaboración propia con base en INE, 2002.

Como se aprecia en la figura 4, hay algunos elementos emergentes de relevancia. El primer caso es el vínculo en doble sentido entre el Gran Concepción y Los Ángeles, destacado con anterioridad.

Otro fenómeno interesante es el efecto de atracción que generan las comunas que son capitales provinciales como Chillán y Los Ángeles. Sin embargo, se detectan también fuertes efectos barrera de éstos hacia comunas aledañas, como San Carlos (-258) en el primer caso y Mulchén (-255) en el segundo. Este efecto de resistencia da cuenta que la población que 


\section{Figura 4}

\section{Principales preferencias migratorias y efectos barrera de las comunas de la región, periodo 1997-2002}

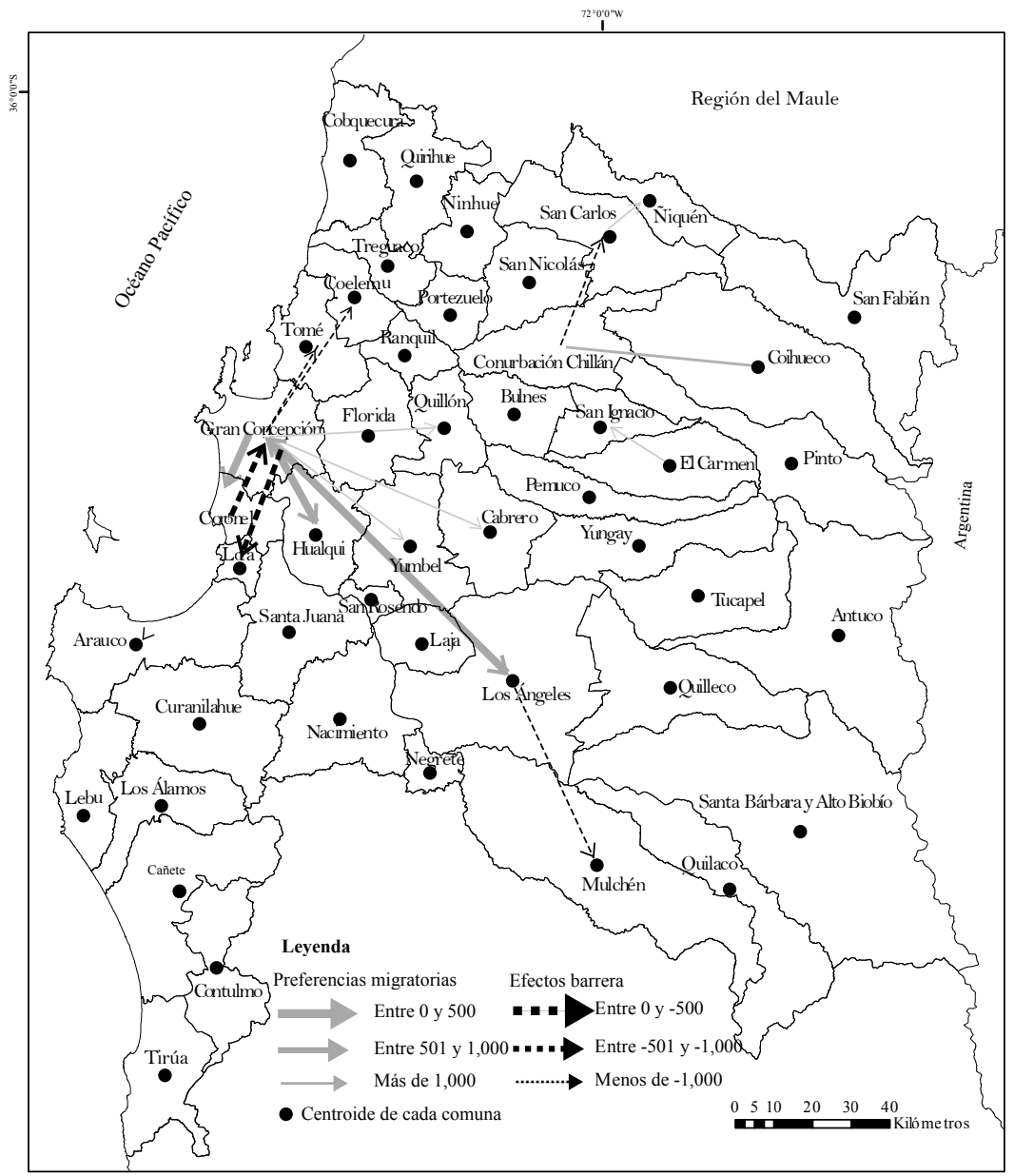

Fuente: elaboración propia con base en INE, 2002.

habita las capitales provinciales tiende a no migrar hacia comunas contiguas. Por el contrario, dichas comunas sí presentan como preferencia migratoria a dichas capitales regionales. Si bien esto parece obvio, otro elemento aparece en el análisis: también la movilidad cotidiana presenta saldos positivos hacia Chillán y Los Ángeles desde sus comunas contiguas; por ejemplo, desde San Carlos conmutan cuatro veces más personas hacia Chillán que de ésta última a la primera comuna (INE, 2002). Es decir, no sólo atraen población de manera permanente con el fenómeno migratorio 
(figuras 3 y 4), sino que también población flotante, lo cual sin duda tiene consecuencias en términos de polarización y dependencia en el espacio.

Así, la relación migración-conmutación tiende en un sentido de manera polarizante, lo cual debería hacer reflexionar en torno a una planificación prospectiva del territorio fomentando dinámicas económicas, sociales, culturales, entre otras, que -idealmente- puedan evitar una concentración excesiva. Futuras investigaciones podrían profundizar en estos aspectos.

Por otro lado, y de manera opuesta, cabe destacar los casos de las comunas de Coronel $(+1,680)$ y Hualqui $(+1,274)$ (ver figura 4$)$ respecto al Gran Concepción. Gracias a la conectividad entregada por el Biotren y un sistema de minibuses, estas comunas presentan una fuerte conmutación por sobre la migración hacia el Gran Concepción. Esto se explica principalmente por su rol de áreas residenciales con viviendas de menor costo asociado a una correcta accesibilidad. Así la relación migraciónconmutación aparece opuesta para el caso de los territorios vinculados a la capital regional.

Sin embargo, esto no ocurre en el caso de Tomé (-273), ya que pese a su proximidad la menor conectividad relativa dificulta, en cierta medida, la interacción, la cual podría ser más potente dada su cercanía, hecho reflejado en el valor residual obtenido por la comuna en cuestión. Aún más fuerte son las barreras para el caso de Lota $(-1,059)$, la cual se muestra como un territorio francamente resistido, pese a su cercanía al Gran Concepción. La razón de esto es que, tras el fin del largo y próspero ciclo carbonífero, la estructura productiva de Lota se caracteriza por una sobrerrepresentación de microempresas, especialmente en el rubro de comercio al detalle (BCN, 2008). Incluso, la actividad económica ahora predominante en Lota (forestal), no tiene incidencia relevante, especialmente respecto de la creación significativa de puestos de trabajo.

De manera global, se aprecia cómo uno de los principales factores para entender los procesos migratorios al interior de la región de Biobío es lo ocurrido con la agricultura cerealera, sector tradicionalmente relevante. En primer lugar, la actividad ha tendido a una consistente baja en la rentabilidad en los últimos años ${ }^{10}$ y la mayor competencia extranjera (González, 2007), lo que ha determinado una disminución significativa a nivel nacional del total de superficie cultivada en el país (de 26,9\% en 1976 a 10,9\% en 2007). Lo anterior ha provocado un significativo proceso de reconversión productiva hacia las plantaciones forestales, generan-

\footnotetext{
${ }^{10}$ Según los cálculos de González (2007: 29-30), los cambios porcentuales absolutos de precios promedio reales a mayoristas, comparando 1990 con 2006 en cifras en pesos chilenos de abril de 2007, son por ejemplo, para el arroz paddy de un $-40 \%$, para el trigo blanco/candeal de un $-21 \%$, para el de la cebada de $-33 \%$ y para el maíz de $-36 \%$, entre otros cultivos tradicionales con bajas significativas.
} 
do un impacto en el empleo por los menores requerimientos de mano de obra de esta última. De hecho, la región del Biobío fue la segunda con mayor reducción de hectáreas cerealeras del país (68,018 hectáreas menos). Por el contrario, las hectáreas con plantaciones forestales prácticamente se duplicaron, pasando de 451,480 en 1976 a 981,756 hectáreas en 2007 (INE, 1976 y 2007a).

\subsection{Perfil de los migrantes}

Una vez establecidos los flujos migratorios al interior de la región del Biobío, resulta necesario profundizar el análisis a través de la elaboración de un perfil de los migrantes y así comprender los tipos de migración predominantes. Para ello, se consideran dos variables: edad (figura 5) y nivel de escolarización de los sujetos (figura 6) según los tres principales territorios con mayor población de la región y los volúmenes observados. ${ }^{11}$ A partir de ello, se obtienen algunas precisiones.

Para el caso de la conurbación Chillán, se detecta una leve diferenciación en el perfil de los migrantes, dependiendo del tamaño de sus comunas de origen. Tal como se señaló con anterioridad, la mayoría de las migraciones hacia Chillán ocurriría respecto de las comunas aledañas, con fuerte componente rural. ${ }^{12}$ En general, la migración desde estas comunas presenta un importante grupo de migrantes entre los 15 y los 19 años, en buena medida en búsqueda de servicios educacionales secundarios y especialmente terciarios. El grupo de migrantes entre los 20 y los 24 años también resulta numeroso en la migración desde estas comunas, predominantemente con un bajo nivel educacional (gran parte de ellos sólo con estudios básicos), lo que da indicios de otro tipo de migración (laboral). En el caso de los migrantes con estudios secundarios dentro de este grupo (minoritarios por cierto), los resultados pueden dar cuenta también de un retraso en el ingreso a la educación superior, lo que debe ser corroborado por estudios de campo.

Por el contrario, la población que migra desde las comunas de mayor población, como Los Ángeles o el Gran Concepción hacia Chillán, tienden a ser personas de mayor edad, en un rango que fluctúa entre los 20 y los 30 años y con mayor cantidad de años de escolaridad. Al considerar la oferta existente en estos centros urbanos, especialmente en el Gran Concepción, la migración por motivos educacionales debiera ser mucho menor que en los casos anteriores (puesto que la capital regional ofrece mayores plazas y diversidad). En efecto, entre los migrantes desde el Gran

\footnotetext{
${ }^{11}$ Por el bajo volumen migratorio expresado por la comuna de Lebu no se considera en el análisis.

${ }^{12}$ Según el censo 2002, numerosas comunas de la provincia de Nuble presentan sobre 60\% de hogares rurales, e incluso algunas bordean 70\% (Coihueco, San Ignacio y San Nicolás).
} 
Concepción predominan los sujetos con estudios superiores, por lo que podría tratarse de migraciones por motivos laborales, o por búsqueda de una mejor calidad de vida. Desde Los Ángeles, el perfil de los migrantes se muestra más homogéneo entre distintos niveles de escolaridad, lo que hace suponer la presencia de varios tipos de motivaciones (estudios, laborales, calidad de vida, etcétera).

Dentro de este análisis general, emerge un caso particular: los migrantes desde la comuna de Pinto hacia Chillán se concentran en un rango etario relativamente acotado (joven y adulto), caracterizado además por un predominio de personas sin estudios superiores. Tal como se muestra en la figura 5, en comparación a las otras comunas migrantes a Chillán, desde Pinto la población se desplaza relativamente más adulta.

\section{Figura 5}

\section{Perfiles de edades migrantes en el Gran Concepción, conurbación Chillán y comuna de Los Ángeles, periodo 1997-2002}

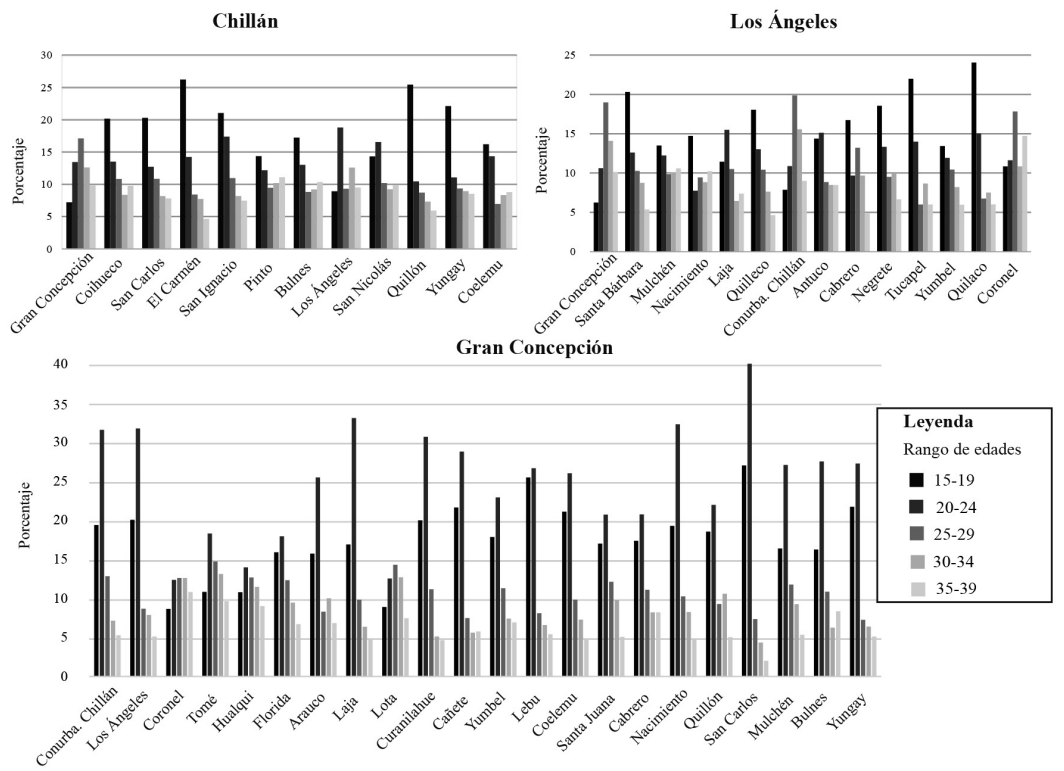

Fuente: elaboración propia con base en INE, 2002.

Para el caso de Los Ángeles, el perfil del migrante también está relacionado con el tamaño de la comuna de origen. En el caso de las comunas pequeñas aledańas (como Quilleco, Negrete y Nacimiento, entre otras), la mayoría de los migrantes se concentra entre los 15 y los 19 ańos, con el predominio de personas con nivel educacional básico (figuras 5 y 6 ). Al igual que en la provincia de Nuble, debido a los porcentajes de rura- 
lidad existentes (en torno a 40\%, destacando Antuco y Santa Bárbara con casi $60 \%$ ), existiría un margen potencial de migración campo-ciudad a desarrollarse. Si bien la migración hacia Los Ángeles desde las comunas con mayor población presenta un perfil superior en cuanto a formación académica de las personas (en comparación a las comunas de menor población), no logra ejercer la atracción de los grupos profesionales en la magnitud que lo hace Chillán. Un caso particular es el de Coronel,,$^{13}$ en el cual el perfil etario de sus migrantes es similar al de los territorios de mayor población. Sin embargo, en términos educacionales, difiere de éstos al predominar las personas con formación básica y media. ${ }^{14}$

\section{Figura 6}

Perfil tipo de educación migrantes en el Gran Concepción, conurbación Chillán y comuna de Los Ángeles, periodo 1997-2002

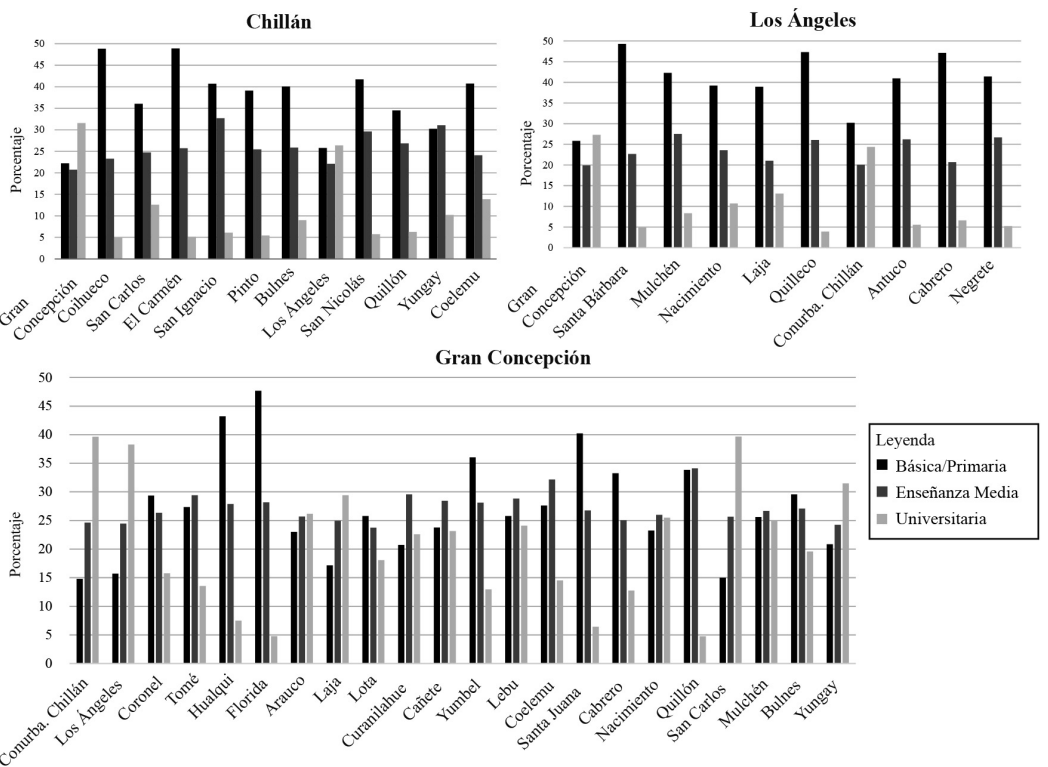

Fuente: elaboración propia con base en INE, 2002.

Finalmente, para el Gran Concepción existe una mayor variedad de perfiles de migrantes. De modo general, y contrario a las comunas precedentes, la población que migra a este espacio metropolitano se concentra

${ }^{13}$ El cual no queda expresado en la figura 6 dado que sus valores eran más bajos que los otros casos sí destacados. Al ser una figura acotada, se buscó graficar la proporción de migrantes en comunas con menor edad.

${ }^{14}$ Debido a la importancia relativa de los datos y alcances de la investigación, en la figura 6 no está expresado el nivel educacional de los migrantes desde Coronel a Los Ángeles. 
entre los 20 y los 24 años (figura 5). Destaca un porcentaje relevante en el tramo de población menor de 20 años, salvo el caso de Coronel, cuya migración es bastante homogénea en cuanto a los rangos etarios. Uno de los factores importantes a considerar es la gran oferta educacional superior existente en el Gran Concepción y las plazas laborales disponibles. Cabe destacar el caso de San Carlos, comuna en que $41 \%$ de la población que migra hacia el Gran Concepción oscila entre 20 y 24 años, siendo el porcentaje más elevado en todos los tramos de las comunas analizadas (figura 6). Además, un alto porcentaje de ellos presenta estudios universitarios $(39,6 \%)$. Es relevante señalar que, a pesar de su cercanía con Chillán, la migración de profesionales jóvenes hacia el Gran Concepción es mucho más significativa.

Respecto al tipo de enseñanza que poseen los migrantes en otras comunas hacia el Gran Concepción, destacan los casos de Chillán y Los Ángeles, con mayor proporción de personas con estudios universitarios, seguidos de San Carlos, Yungay y Laja. Estos últimos resultan bastante particulares e interesantes, porque se trata de comunas con baja población. La preferencia de migración profesional de estas tres se relaciona con el efecto barrera detectado respecto de Los Ángeles y en menor medida Chillán (ver figura 3), lo cual explica la migración al Gran Concepción. Pese a la cercanía con las capitales provinciales respectivas, en estos segmentos educados la migración se produce preferentemente hacia la capital regional.

Los migrantes al Gran Concepción desde otras comunas presentan un perfil educacional predominantemente con estudios básicos. Sin embargo, en algunas comunas aledañas como Coronel, Lota y Tomé, la proporción de migrantes con estudios de enseñanza media también es importante y prácticamente equivalente a la anterior, lo cual nos dice que la migración podría estar asociada a un factor laboral o para proseguir estudios técnicos o universitarios.

En este sentido, la oferta universitaria muestra una concentración sustantiva en el Gran Concepción. Si bien la situación es esperable, es interesante destacar que, del conjunto de universidades analizadas, ${ }^{15} 74 \%$ de las carreras de pregrado impartidas en la región se concentran en el Gran Concepción, contrastando para el caso de Los Ángeles que presenta $15 \%$ y Chillán $11,2 \%$. Respecto a los postgrados, sobre $75 \%$ de los

\footnotetext{
${ }^{15}$ Se realizó un catastro de las carreras de pregrado impartidas por las siguientes instituciones: Universidad del Bío-Bío, Universidad de Concepción, Universidad del Desarrollo, Universidad San Sebastián, Universidad de Las Américas, Universidad Católica Santísima de Concepción, Universidad de la República, Universidad Andrés Bello, Universidad Santo Tomás, Universidad Bolivariana, Universidad Técnico Federico Santa María y Universidad Tecnológica de Chile INACAP. Fueron consideradas carreras tanto profesionales como técnicas según la información de los planteles universitarios.
} 
programas de magister y $100 \%$ de la formación de doctorados se concentran en el Gran Concepción.

\section{Conclusiones}

A partir de los resultados obtenidos del estudio, es posible concluir varios procesos. En primer lugar, existe una consolidación del Gran Concepción como nodo articulador del espacio y centro captador de los mayores flujos migratorios. Lo anterior no es sorprendente, considerando su tamaño de población y la diversidad de servicios e instituciones desconcentradas del Estado que se localizan en dicho espacio.

Posteriormente, Los Ángeles y Chillán se constituyen en nodos relevantes en la recepción de migrantes. A lo lejos se encuentra la capital de la provincia de Arauco (Lebu), la cual, pese a ostentar un rango en la división político-administrativa y teóricamente presentar un liderazgo, se ve desfavorecida frente a la comuna de Los Álamos.

En segundo lugar, se detecta una estructura espacial diferenciada en la migración en la región del Biobío. Por un lado, la población con alto nivel educacional tiende a migrar al Gran Concepción, especialmente desde Los Ángeles, Chillán, Laja, San Carlos y Yungay (pese a la proximidad de las tres últimas con su respetiva capital provincial). Por otra parte, la población joven con baja calificación (salvo la correspondiente a la Provincia de Concepción), tiene una preferencia migratoria hacia las capitales provinciales. Varios factores pueden incidir en esta situación, como las oportunidades laborales, el costo del suelo, acceso a vivienda y redes familiares y sociales que facilitarían tal migración.

Así, un tercer elemento entra en discusión: las migraciones se vinculan con las variaciones en la estructura productiva, la cual incide directamente en la demanda laboral y el perfil de quienes acceden a ella. Por lo tanto, es posible afirmar que las comunas rurales de la región estarían en su gran mayoría expulsando población joven hacia las capitales provinciales (salvo Concepción) dado un contexto productivo-estructural de la economía regional. En efecto, la disminución de la rentabilidad de los cultivos tradicionales y la reconversión productiva hacia las plantaciones forestales, con menor requerimiento de mano de obra, ha sido un factor importante para explicar la presión migratoria en segmentos jóvenes de poblaciones rurales, considerando además otros factores como el cambio en las expectativas de vida de los jóvenes rurales (Arteaga, 2000).

En cuarto lugar, los resultados han reflejado que al interior de la región del Biobío, la provincia de Nuble presenta características que sugieren cierto aislamiento respecto del resto del espacio, reflejado en bajas magnitudes de interacción con las comunas ajenas a la provincia. A medida 
que se cuente con datos más recientes, otras investigaciones podrán profundizar y reforzar los fenómenos acá planteados.

Finalmente, y en relación con el Modelo Gravitacional, queda demostrada su utilidad para la comprensión de la estructura resultante de la interacción espacial y, potencialmente, para otros procesos como movilidad entre diferentes territorios.

Sin embargo, hay que considerar las limitaciones de este tipo de modelos, dado que obedecen a una simplificación de una realidad naturalmente compleja. Por lo mismo, a ellos se deben ir incorporando otros factores que ayuden a comprender los procesos migratorios al interior de la región y que permitan proyectar flujos en el futuro. Esto de acuerdo con distintos escenarios regionales que se puedan ocurrir acorde a nuevas políticas públicas (por ejemplo descentralizadoras o propulsoras de clúster productivos), creación de empleo y realidades socio-económicas de la población, entre otros. Sin duda lo anterior se convierte en un desafío para investigaciones futuras a realizar. Vinculado a ello, resultaría fundamental y oportuno incorporar el enfoque aquí estudiado en los instrumentos de planificación territorial, puesto que permite generar un mejor diagnóstico y conocimiento del territorio, además de elevar las metodologías que sustentan dichas herramientas.

\section{Fuentes consultadas}

Alm, James y John Winters (2009), "Distance and intrastate college student migration", Economics of Education Review, 28 (6), Elsevier, Londres pp. 728-738.

Anderson, James y Eric Van Wincoop (2003), "Gravity with gravitas: a solution to the border puzzle", American Economic Review, 93 (1), American Economic Association, Pittsburgh, pp. 170-192.

Aroca, Patricio, Geoffrey Hewings y Jimmy Paredes (2001), "Migración interregional y el mercado laboral en Chile 1977-1982 y 19821992", Cuadernos de economía, 38 (115), Pontificia Universidad Católica de Chile, Santiago, pp. 321-345.

Aroca, Patricio (2004), "Migración interregional en Chile modelos y resultados 1987-2002”, Notas de población, 78 (31), Cepal, Santiago, pp. 97-154. 
Arteaga, Catalina (2000), Modernización agraria y construcción de identidades en Chile, Plaza y Valdés-FLACSO México-CEDEM, México.

BCN (Biblioteca del Congreso Nacional de Chile) (2008), "Reporte Estadístico Comunal. Lota. Sistema Integrado de Información Territorial”, Biblioteca del Congreso Nacional, Valparaíso.

Borsdorf, Axel, Rafael Sánchez y Carla Marchant (2008), “Aprendiendo de los errores. La necesidad de cambios a la política nacional de vivienda en ciudades intermedias chilenas", Scripta Nova, Revista electrónica de geografía y ciencias sociales, XII (270), Universidad de Barcelona, Barcelona.

Busso, Gustavo (2007), “Argentina, Bolivia, Brasil y Chile: pobreza y efectos sociodemográficos de la migración interna a inicios del siglo XXI", Notas de Población, 34 (84), Cepal, pp. 53-85.

Camagni, Roberto (2005), Economía urbana, Antoni Bosch, Barcelona.

Campaniello, Nadia (2014), "The causal effect of trade on migration: Evidence from countries of the Euro-Mediterranean partnership", Labour Economics, 30, Elsevier, pp. 223-233.

Ceglowski, Janet (2006), "Does gravity matter in a service economy?", Review of world economics, 142 (2), Springer, Berlin Heidelberg, pp. 307-329.

Chen, Yanguang (2015), "The distance-decay function of geographical gravity model: Power law or exponential law?", Chaos, Solitons and Fractals, 77, Elsevier, pp. 174-189.

Chen, Yanguang y Fahui Wang (2008), "Fourier analysis of an expanded gravity model for spatio-temporal interactions", Physics and Society, 10 (3), American Physical Society, New York, pp. 325-347.

Curry, Leslie (1972), "A spatial analysis of gravity flows", Regional Studies, 6 (2), The Regional Studies Association, Routledge, Seaford, pp. 131-147.

Di Filippo, Armando y Rosa Bravo (1977), "Los centros nacionales de desarrollo y las migraciones internas en América Latina: un estu- 
dio de casos, Chile", Revista Latinoamericana de Estudios Urbano Regionales, 5 (14), Pontificia Universidad Católica de Chile, Santiago, pp. 67-101.

Durán-Fernández, Roberto y Georgina Santos (2014), "Gravity, distance, and traffic flows in Mexico", Research in Transportation Economics, 46, Elsevier, Atlanta, pp. 30-35.

Faura, Úrsula y Juan Gómez (2001), "Modelos migratorios: una revisión”, Revista Asturiana de economía, 1 (21), Universidad de Oviedo, Oviedo, pp. 209-235.

Fukunari, Kukunari y Lee Hyun-Hoon (2006), "The gravity equation in international trade in services", Review of World Economics, 142 (1), Springer, Berlin Heidelberg, pp. 92-121.

González, Jorge (2007), "Sector agropecuario de la VIII Región del BíoBío", documento de trabajo, Departamento de Economía Agraria, Centro Regional de Investigación Quilamapu INIA, Santiago.

Groschet, Tobias, Franz Rothlauf y Armin Heinzl (2007), "Gravity models for airline passenger volume estimation", Journal of Air Transport Management, 13 (4), Elsevier, London, pp. 175-183.

Haynes, Kingsley y Stewart Fotheringham (1984), Gravity and spatial interaction models, Sage Publications, London.

Hall, Peter y Kathy Pain (2006), The polycentric metropolis. Learning from mega-city regions in Europe, Earthscan, London.

Hidalgo, Rodrigo y Hugo Zunino (2011), "La urbanización de las áreas periféricas en Santiago y Valparaíso: el papel de las relaciones de poder en el dibujo de la geografía sociorresidencial", Revista Latinoamericana de Estudios Urbano Regionales, 37 (111), Pontificia Universidad Católica de Chile, Santiago, pp. 79-105.

Hidalgo, Rodrigo y Michael Janoschka (2014), La ciudad Neoliberal. Gentrificación y exclusión en Santiago de Chile, Buenos Aires, Ciudad de México y Madrid, Serie GEOlibros, Pontificia Universidad Católica de Chile. 
Hurtado, Carlos (1966), Concentración de población y desarrollo económico: el caso chileno, Instituto de Economía UCH, Santiago.

INE (Instituto Nacional de Estadísticas de Chile), (2012) "Censo nacional de población año 2012", base de datos, Gobierno de Chile, Santiago.

INE (Instituto Nacional de Estadísticas de Chile) (2007a), "VII Censo Nacional Agropecuario y Forestal. Año agrícola 2006-2007. Total país", Departamento de Demografía, Geografía y Censos, Gobierno de Chile, Santiago.

INE (Instituto Nacional de Estadísticas de Chile) (2007b), "Migraciones regionales 1992-2002", documento de trabajo, Departamento de Demografía, Geografía y Censos, Gobierno de Chile, Santiago.

INE (Instituto Nacional de Estadísticas de Chile) (2004), "Migración inter e intra regional", documento de trabajo, Dirección regional del Biobío, Gobierno de Chileno, Santiago.

INE (Instituto Nacional de Estadísticas de Chile) (2002), "Censo nacional de población año 2002", base de datos, Gobierno de Chile, Santiago.

INE (Instituto Nacional de Estadísticas de Chile) (1992), "Censo Nacional de población año1992", base de datos, Gobierno de Chile, Santiago.

INE (Instituto Nacional de Estadísticas de Chile) (1982), "Censo Nacional de población año1982”, base de datos, Gobierno de Chile, Santiago.

INE (Instituto Nacional de Estadísticas de Chile) (1976), "V Censo Nacional Agropecuario. Año agrícola 1975-1976. Total país”, Gobierno de Chile, Santiago.

Karemera, David, Victor Iwuagwu y Bobby Davis (2000), “A gravity model analysis of international migration to North America", Applied Economics, 32 (13), Routledge, Kentucky, pp. 1745-1755. 
Lattes, Alfredo (2001), "Población urbana y urbanización en América Latina", en Fernando Carrión F. (ed.), La ciudad construida, urbanismo en América Latina, Flacso, Quito, pp. 49-76.

Lewer, Joshua y Hendrik Van den Berg (2008), "A gravity model of immigration", Economics Letters, 99 (1), Elsevier, Amsterdam, pp. 164-167.

Martínez, Jorge (ed.) (2008), América Latina y el Caribe: migración internacional, derechos humanos y desarrollo, Cepal, Santiago.

Morley, Clive, Jaume Rosselló y María Santana-Gallego (2014), "Gravity models for tourism demand: theory and use", Annals of Tourism Research, 48, Elsevier, London, pp. 1-10.

Niedercorn, John y Burley Bechdolt (1969), "An economic derivation of the gravity law of spatial interactions", Journal of Engineering Science, 9 (2), Wiley, Chichester, pp. 273-282.

Olsson, Gunnar (1965), Distance and human interaction: a review and bibliography, Regional Science Research Institute, Philadelphia.

Precedo, Andrés, Javier Orosa y Alberto Iglesias (2010), "De la planificación estratégica al marketing urbano: hacia la ciudad inmaterial", Revista Latinoamericana de Estudios Urbano Regionales, 36 (108), Pontificia Universidad Católica de Chile, Santiago, pp. 5-27.

Pumain, Denise y Thérèse Saint-Julien (2001), Analyse Spatiale. Les Interactions, Armand Colin, Paris.

Raczynski, Dagmar (1982), "Éxodo rural en Chile”, en Raczynski, Dagmar, Determinantes del éxodo rural: importancia de factores del lugar de origen, Chile, 1965-1970, Cieplan, Santiago, pp. 61-104.

Rodrigo, Luis y Miguel Atienza (2014), "Migración y representaciones regionales: discursos sobre la Región de Antofagasta”, Revista Latinoamericana de Estudios Urbano Regionales, 40 (120), Pontificia Universidad Católica de Chile, Santiago, pp. 159-181.

Rodríguez, Jorge y Gustavo Busso (2009), Migración interna y desarrollo en América Latina entre 1980 y 2005. Un estudio comparativo con perspectiva regional basado en siete países, Cepal, Santiago. 
Rodríguez, Jorge y Daniela González (2006), “Redistribución de la población y migración interna en Chile: continuidad y cambio según los últimos cuatro censos nacionales de población y vivienda", Revista de Geografía Norte Grande, 1 (35), Pontificia Universidad Católica de Chile, Santiago, pp. 7-28.

Rodríguez, Juan y Medina, Patricio (2011), "Reconversión, daño y abandono en Lota", Atenea, 1 (504), Universidad de Concepción, Concepción, pp. 147-176.

Roy, John (2004), Interaction modelling: a regional science context, Springer, Berlin.

Sandoval, Carlos (2011), De subterra a subsole. El fin de un ciclo, Quimantú, Chile.

Schiappacasse, Paulina, Miguel Contreras y Carlos Fuensalida (2001), "Migraciones internas hacia la Región Metropolitana de Santiago de Chile: una comparación con planteamientos teóricos", Investigaciones Geográficas, 1 (35), Universidad de Chile, Santiago, pp. 1-25.

Sistema de Información Geográfica ArcGis 10, "Distancias calculadas utilizando Análisis de Redes, en base a Red Vial y cabeceras comunales", <http://servicios.vialidad.cl/Distancias/Distancias. asp>, 5 de agosto de 2013.

Solimano, Andrés (2008), Migraciones internacionales en América Latina. Booms, crisis y desarrollo, Fondo de Cultura Económica, Santiago.

Stewart, John Q. (1948), "Demographic gravitation: evidence and applications", Sociometry, 11 (1/2), American Sociological Association, Washington, pp. 31-58.

Thomas, Reginald y Richard Huggett (1980), Modelling in geography a mathematical approach, Harper \& Row, Publishers, London.

Veltz, Pierre (2005), Mondialisation, villes et territoires: l'économie d'archipel, Presses universitaires de France, Paris. 
Wilson, Alan (1971), "A family of spatial interaction models and associated developments", Environment and Planning, 3 (1), Sage Publications, Thousand Oaks, pp. 1-32.

Zelinsky, Wilbur (1971), "The hypothesis of the mobility transition", Geographical Review, 61 (2), The American Geographical Society, San Diego, pp. 219-249.

Recibido: 5 de noviembre de 2015. Corregido: 28 de diciembre de 2015. Aceptado: 20 de enero de 2016.

Francisco Maturana-Miranda. Chileno. Doctor en Planificación Territorial y Dinámicas del Espacio de la Universidad Paris-Sorbonne, académico en el Departamento de Geografía de la Universidad Alberto Hurtado (Chile). Su línea de investigación es el análisis y comprensión de los sistemas de ciudades bajo el contexto de la planificación territorial. Entre sus últimas publicaciones destacan, en coautoría: "Trayectoria, evolución y estructura del sistema de ciudades. El caso de la región del Biobío (Chile)", Cuadernos de Geografía: Revista Colombiana de Geografía, 25 (2), Universidad Nacional de Colombia, Colombia, pp. 39-58 (2016); "Ciudades intermedias en Chile: definición de un territorio en transición”, Revista Planeo, 27, Pontificia Universidad Católica de Chile, Santiago, <http://revistaplaneo.uc.cl/2016/04/04/ciudades-intermediasen-chile-definicion-de-un-territorio-en-transicion/>, (2016); Ciudades intermedias en Chile: Territorios olvidados, RIL Editores, Santiago de Chile (2015).

Andrés Rojas-Böttner. Chileno. Magíster en Estudios Sociales y Políticos Latinoamericanos, por la Universidad Alberto Hurtado. Investigador del Instituto Chileno de Estudios Municipales de la Universidad Autónoma de Chile. Su línea de investigación está orientada a la Historia económica regional, formación de Estado y desarrollo territorial en perspectiva histórica. Entre sus publicaciones destacan: "Mecanización agrícola y fomento estatal en Chile (1942-1973)", América Latina en la Historia Económica, 23 (3), Instituto de Investigaciones Dr. José María Luis Mora, México (2016); en coautoría, "Trayectoria, evolución y estructura del sistema de ciudades. El caso de la región del Biobío (Chile)", Cuadernos de Geografia: Revista Colombiana de Geografia, 25 (2), Universidad Nacional de Colombia, Bogotá, pp. 39-58 (2016); "Atacama Rebelde: Im- 
pugnación constituyente al centralismo (1859)”, en Esteban Valenzuela (ed.), Territorios Rebeldes, Editorial Universidad Alberto Hurtado, Santiago, pp. 82-107 (2015); "Evolución histórica de las ciudades intermedias en el siglo XX: crecimiento, jerarquía y funcionalidad”, en Maturana Francisco y Andrés Rojas, Ciudades Intermedias en Chile: territorios olvidados, Editorial RIL, Santiago, pp. 43-74 (2015).

David Poblete-López. Chileno. Magister en Hydroinformatics and Water Management - EUROAQUAE. Universidades: Newcastle University (Gran Bretaña), Budapest University of Economics and Technology (Hungría), University of Nice-Sophia Antipolis (Francia). Se desempeña como Académico en la Escuela de Ingeniería de la Universidad de Valparaíso. Sus líneas de investigación reciente han estado orientadas al estudio del cambio climático e impactos en la ciudad, participando en proyectos CORFO-Innova y Fondef. Entre sus últimas publicaciones destacan, en coautoría: "Exploring possible connections between hydrologic extreme events and climate change in central south Chile", Hydrological Sciences Journal, 58 (8), Routledge, Seaford, pp. 1598-1619 (2013); “Using a gridded global dataset to characterize regional hydroclimate in Central Chile", Journal of Hydrometeorology, 14 (1), American Meteorological Society, Boston, pp. 251-265 (2013); "Water-food-energy nexus in Chile: the challenges due to global change in different regional contexts", Water International, 40 (5-6), Routledge, Seaford, pp. 839-855 (2015). 